Article

\title{
Energy-Dependent RBS Channelling Analysis of Epitaxial ZnO Layers Grown on ZnO by RF-Magnetron Sputtering
}

\author{
Florian Wittkämper ${ }^{1,2}$, André Bikowski ${ }^{3}$, Klaus Ellmer ${ }^{3}$, Konrad Gärtner ${ }^{1}$ and Elke Wendler ${ }^{1, *}$ \\ 1 Friedrich-Schiller-Universität Jena, Institut für Festkörperphysik, Max-Wien-Platz 1, 07743 Jena, Germany; \\ florian.wittkaemper@leibniz-ipht.de (F.W.); konrad.gaertner@uni-jena.de (K.G.) \\ 2 Leibniz-Institut für Photonische Technologien, Albert-Einstein-Straße 9, 07745 Jena, Germany \\ 3 Helmholtz-Zentrum Berlin für Materialien und Energie GmbH (HZB), Institute for Solar Fuels, \\ Hahn-Meitner-Platz 1, 14109 Berlin, Germany; bikowski@out-ev.de (A.B.); ellmer@out-ev.de (K.E.) \\ * Correspondence: elke.wendler@uni-jena.de; Tel.: +49-3641-947330
}

Received: 30 April 2019; Accepted: 30 May 2019; Published: 4 June 2019

\begin{abstract}
The transparent conducting oxides $\mathrm{ZnO}$ and $\mathrm{ZnO}: \mathrm{Al}$ are interesting materials for a wide range of applications. Several of these applications need a large area, single crystalline, and specially doped thin layers. A common technique for the fabrication of those layers is RF (radio frequency) -magnetron sputtering. The investigation of the crystal quality of such layers requires methods of analysis that are destruction free and that are able to obtain information about the concentration and type of defects versus depth. One such option is the Rutherford backscattering spectroscopy (RBS) in channelling mode. In this work, we exploit the channelling effect and its energy dependence, which are sensitive to the type of defects. By using appropriate software and measuring RBS channelling spectra with different beam energies, we were able to determine the depth distribution of point defects and dislocation loops. The presence of dislocation loops was proven using other previously applied analysis methods. The main advantage of RBS in channelling mode is the quantification of point defects, which can be important for defining the electrical and optical properties of such layers. The technique demonstrated is applicable to other defective crystals or thin crystalline layers.
\end{abstract}

Keywords: ZnO:Al; RBS channelling; structural characterisation

\section{Introduction}

Zinc oxide $(\mathrm{ZnO})$ belongs to a group of transparent conductive oxides, which are a unique class of materials. It exhibits both high transparency in the visible and near infrared spectral range and high electronic conductivity. Additionally, $\mathrm{ZnO}$ is abundant-therefore cheap-and non-toxic. This makes the material interesting for a wide range of applications such as thin film solar cells, architectural glass coatings and flat-panel displays (see, e.g., [1] and references therein). Polycrystalline $\mathrm{ZnO}$ is sufficient for many applications, whereas the development of $\mathrm{ZnO}$-based optoelectronic applications requires epitaxial monocrystalline thin films (see, e.g., [2] and references therein). Among other techniques for thin film deposition, RF-magnetron sputtering is attractive, especially due to its high deposition rates at relatively low substrate temperatures [3]. The substrate type and deposition parameters, such as the mode and temperature of deposition, affect the crystallinity and the defect density of the layers, and therefore their electrical and optical properties.

Many techniques are applied for the analysis of defects in thin films. In this work, Rutherford backscattering spectrometry (RBS) in channelling configuration is used [4,5]. Herein, a well collimated mono-energetic beam of light ions, usually helium (He) ions or protons, impinges on a target. Ions are scattered into the half-space above the target. A detector measures the energy of the backscattered 
ions. The position of the detector determines the backscattering angle (see Figure 1). The signal of the detector is electronically processed and the number of ions is counted as a function of their energy. Thus, the result of the RBS measurement is the number of ions scattered into the detector as a function of the ion energy, which is called the RBS spectrum. This method is very commonly used, especially for studying ion-beam induced damage in crystalline solids [6]. It is briefly described in Section 3. The advantages of this method are that samples do not require special preparation and RBS is nearly destruction free. By exploiting the channelling effect, the depth distribution of lattice defects can be extracted from the measured RBS spectra. Additionally, further information about the dominate type of defects can be obtained when spectra are measured under different conditions. Those conditions are different temperatures or different energies of the analysing ions.

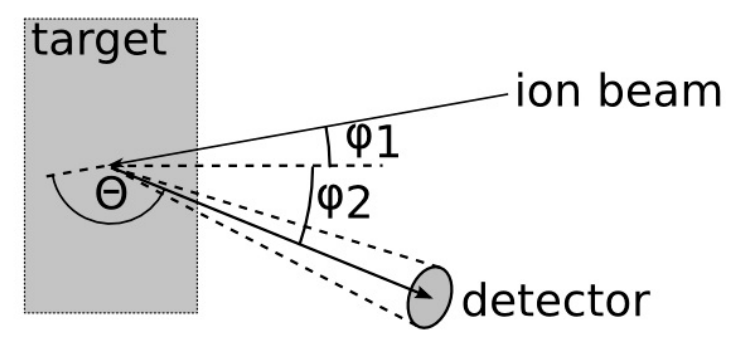

Figure 1. Schematic representation of Rutherford backscattering spectrometry (RBS). The angles of incidence and exit of the analyzing ions are $\varphi_{1}$ and $\varphi_{2}$, respectively. $\Theta$ is the backscattering angle.

In this work, the capability of energy-dependent RBS in channelling mode is demonstrated for epitaxial $\mathrm{ZnO}$ layers sputtered onto single-crystalline $\mathrm{ZnO}$ substrates. The existence of point defects and dislocation loops well describes the channelling RBS spectra measured for the various beam energies applied. The concentration and depth distribution of both types of defects were determined.

\section{Experimental Conditions}

$\mathrm{ZnO}$ and Aluminium-doped $\mathrm{ZnO}$ ( $\mathrm{ZnO}: \mathrm{Al})$ layers grown homoepitaxially on the $\mathrm{Zn}$-terminated surface of undoped $\mathrm{ZnO}<0001>$ substrates were investigated by energy-dependent RBS in channelling configuration.

The layers were prepared by RF-magnetron sputtering at a constant power of $50 \mathrm{~W}$ and a substrate temperature of $300{ }^{\circ} \mathrm{C}$ (for more details of the experimental set-up and the deposition procedure see [7]). Aluminium doping is obtained by using a special ceramic target $\left(\mathrm{ZnO} / \mathrm{Al}_{2} \mathrm{O}_{3} 98 / 2 \mathrm{wt} . \%\right)$. The $\mathrm{Al}$ content of the films, measured by RBS, is about ( $2 \pm 0.2)$ at.\% [8]. The layer thickness was measured with a surface profilometer [9]. Table 1 presents the various deposition frequencies applied, and the composition and thickness of the investigated layers.

Table 1. Overview of the investigated $\mathrm{ZnO}$ samples and their parameters.

\begin{tabular}{cccc}
\hline Sample & $\begin{array}{c}\text { Discharge Frequency } \\
\text { (MHz) }\end{array}$ & $\begin{array}{c}\text { Al Fraction } \\
\text { (at. \%) }\end{array}$ & $\begin{array}{c}\text { Thickness } \\
\text { (nm) }\end{array}$ \\
\hline AB5897 & 13.56 & 0 & 565 \\
AB6008 & 13.56 & 2 & 735 \\
AB6200 & 27.12 & 0 & 1215 \\
AB6227 & 27.12 & 2 & 785 \\
\hline
\end{tabular}

The RBS measurements were carried out with He ions at energies of 1.4, 2.0, 2.5 and $3.0 \mathrm{MeV}$. The backscattered ions were registered with the detector placed at $170^{\circ}$ with respect to the direction of the incident ion beam (see Figure 1). An aligned spectrum is obtained when the ions penetrate into the crystal along a low-index axis. Random spectra were taken with the samples being tilted 5 degrees off axis and rotated around an axis perpendicular to the sample surface. The minimum 
yield $\chi_{\min }$ is calculated from the energy spectra of backscattered ions measured in aligned $\left(Y_{\mathrm{al}}\right)$ and in random $\left(Y_{\text {ra }}\right)$ direction with $\chi_{\min }=Y_{\text {al }} / Y_{\text {ra }}$. Energy-to-depth conversion was performed using common SRIM (Stopping and Range of Ions in Matter) energy loss data [10]. The minimum yield $\chi_{\min }$ versus depth $z$ (i.e., the $\chi_{\min }(z)$ spectra) obtained for the different beam energies were used in order to obtain information about the type and concentration of defects within the sputtered $\mathrm{ZnO}$ layers. With respect to RBS in channelling configuration, all defects in a crystal manifest as displaced lattice atoms. In this work, the DICADA code [11] and a previous version DICADA1 [12] are applied for defect analysis, which are based on the discontinuous model of dechannelling [13,14]. With the DICADA code, $\chi_{\min }$ spectra can be calculated as a function of depth $z$ for a given relative concentration of displaced lattice atoms $n_{\mathrm{da}}(z)$ for compound and single crystals. Conversely, the relative concentration of displaced lattice atoms versus depth, $n_{\mathrm{da}}(z)$, can be calculated from the measured minimum yield $\chi_{\min }(z)$. The program version DICADA1 is applicable only to elementary targets but includes both point defects and extended defects. Therefore, this version was used to quantify the amount of point defects and dislocation loops in the epitaxial layers by fitting calculated $\chi_{\min }(z)$ spectra to the measured ones. For this, the binary material $\mathrm{ZnO}$ has to be approximated by an elementary one with mass and atomic number taken as a mean value of that of the two elements of the original crystal. The usability of this approach has already been demonstrated for defect studies of ion-implanted SiC [15]. For the calculations we used an average atomic number of $Z_{\mathrm{av}}=19$ and an average mass of $m_{\mathrm{av}}=40.69 \mathrm{u}$. The Debey temperature was taken to be 416 K [16] and the lattice parameters are $a=3.2498 \AA$ and $c=5.2066 \AA$ (JCPDS 36-1451).

\section{RBS Method}

As already mentioned above, an aligned RBS spectrum is obtained when the ions enter a monocrystalline solid in the direction of a low-index axis. The atoms of the crystal are arranged in rows which form channels along the low-index axis. Within these channels the ions perform a guided movement due to the periodic atomic potentials-this is called the channelling effect. It occurs if the angle $\Psi$ between the ion velocity and the atomic rows is below a critical value $\Psi_{c}$. The consequence of channelling is a reduction in the probability of the occurrence of direct backscattering events. Thus, a significant decrease in the yield of backscattered ions is observed in comparison to the yield measured in random direction. Defects in crystals appear as atoms being displaced from their original lattice sites. Direct backscattering takes place when an ion gets backscattered in a single collision with a displaced lattice atom. Additionally, the displaced lattice atoms disturb the movement of the ions within the channels, resulting in an increase of $\Psi$. When $\Psi$ exceeds the critical angle $\Psi_{c}$, the guided movement of the ions is ceased, and eventually backscattering takes place in a further scattering event. This process is called dechannelling. Both processes occur simultaneously. In the case of direct backscattering, backscattering of the ions takes place at the depth at which the displaced lattice atom is located. Backscattering of ions due to dechannelling is the consequence of all defects within the layer the ions traversed up to the depth where the backscattering event takes place. This contribution to the aligned backscattering spectra is called the dechannelling background.

In previous works, it was demonstrated that the contribution of direct backscattering and dechannelling to the aligned RBS spectra depends on the kind of defects [4,5]. Point defects, point defect complexes and amorphous zones (here summarized as point defects) are characterised by uncorrelated-displaced lattice atoms. In this case, the contribution of direct backscattering is significant. Conversely, in the case of extended defects, a correlated displacement of lattice atoms occurs and the contribution of direct backscattering is negligible. This means that the measured aligned RBS spectra are mainly determined by the dechannelling of the analysing He ions. This effect is illustrated in Figure 2, which shows $\chi_{\min }(z)$ spectra calculated for $<001>$ oriented silicon damaged within the first $0.25 \mu \mathrm{m}$ (data taken from [17]). Additionally, the spectrum of the perfect crystal, $\chi_{\text {min }}^{\text {perf }}(z)$, is shown for comparison. Curve A is calculated assuming a Gaussian distribution of randomly-displaced lattice atoms with a maximum concentration of 0.5 at a depth of $0.1 \mu \mathrm{m}$. A clear damage peak due to direct 
backscattering occurs in this case. Behind the damaged layer, the minimum yield does not drop down to the value of the perfect crystal, because of the dechannelling of the ions that passed through the damaged layer. Curve B in Figure 1 was calculated for a $0.25 \mu \mathrm{m}$ thick layer containing a certain amount of dislocation loops. No damage peak is visible in this spectrum. The end of the damaged layer is indicated by the change in the slope of $\chi_{\min }$ with depth $z$ at $0.25 \mu \mathrm{m}$.

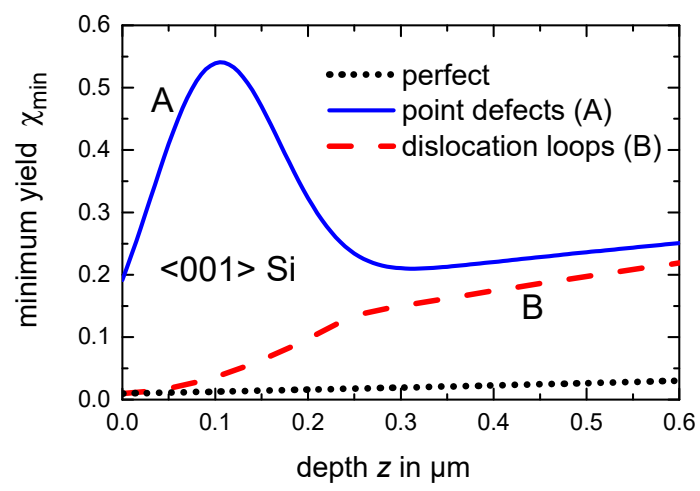

Figure 2. Minimum yield $\chi_{\text {min }}$ versus depth $z$ calculated for $1.4 \mathrm{MeV}$ He ions and $<001>$ Si with different defect states within the first $0.25 \mu \mathrm{m}$ : Gaussian distribution of point defect complexes (solid line, A) and box-like distribution of dislocation loops (dashed line, B). The $\chi_{\min }(\mathbf{z})$ spectrum of undamaged $\mathrm{Si}$ is given as a dotted line for comparison. For details see text.

Theoretical and experimental investigations revealed that the minimum yield $\chi_{\min }$ at a given depth behind a damaged layer depends on the beam energy $E[4,5]$. For the analysis, the dechannelling probability $\gamma$ is relevant with $\gamma=\ln \left[\left(1-\chi_{\min }^{\text {perf }}\right) /\left(1-\chi_{\min }\right)\right]$. In a first approximation and with $1-$ $\chi_{\min }^{\text {perf }} \approx 1$, one obtains $\gamma \approx \chi_{\min }$. A dependence of the minimum yield according to $\gamma \sim E^{\mathrm{K}}$ is obtained for uncorrelated displaced lattice atoms with $k=(-0.5$ to -1$)$ depending on the distribution and concentration of the displaced lattice atoms $[5,18]$. In the case of extended defects, a beam energy dependence of $\gamma \sim E^{0}$ is obtained for stacking faults and $\gamma \sim E^{0.5}$ for dislocation loops [12,19]. That is, the beam energy dependences are distinctly different for uncorrelated and correlated displaced lattice atoms. This effect has been used to obtain information about the kind of defects in ion-implanted crystals by RBS in channelling configuration. In this work, it was applied to sputtered ZnO layers.

\section{Results and Discussion}

Figure 3 depicts the energy spectra of backscattered He ions for all the samples investigated and all the ion energies applied. Oxygen resonances [20] enhance the yield of backscattered ions for beam energies of $2.5 \mathrm{MeV}$ around channel 170, and of $3 \mathrm{MeV}$ around channel 210. The random spectra of $\mathrm{Al}$ doped $\mathrm{ZnO}$ show a slightly reduced $\mathrm{Zn}$ backscattering yield within the deposited layer due to the presences of $\mathrm{Al}$ atoms. For all applied beam energies, this decrease corresponds to an $\mathrm{Al}$ concentration of $2 \%$, which is in agreement with the composition of the sputter target. As a reference for perfect $\mathrm{ZnO}$, aligned spectra were measured on undoped $\mathrm{ZnO}<0001>$ substrates. In Figure 3 it can be seen that the backscattering yield of the sputtered layer is rather high for sample AB5897 (ZnO deposited with $13.56 \mathrm{MHz}$ ). This points to a stronger contribution of direct backscattering than in other layers and indicates the formation of a heavily damaged layer. For the other three $\mathrm{ZnO}$ layers, the backscattering yield in aligned direction is significantly lower, indicating a low level of damage. The spectra are characterised by a strong dechannelling of the analysing He ions. In this case, the end of the sputtered $\mathrm{ZnO}$ layers can be identified by the change in the slope of the spectra with decreasing channel numbers, and therefore decreasing ion energy. For 1.4 MeV He ions, the maximum depth of analysis (backscattering on $\mathrm{Zn}$ atoms only without superposition of backscattering from $\mathrm{O}$ ions) is about $700 \mathrm{~nm}$. This is less than the thickness of most layers (see Table 1). Therefore, the end of the sputtered layers is less visible in the $1.4 \mathrm{MeV}$ He spectra. 


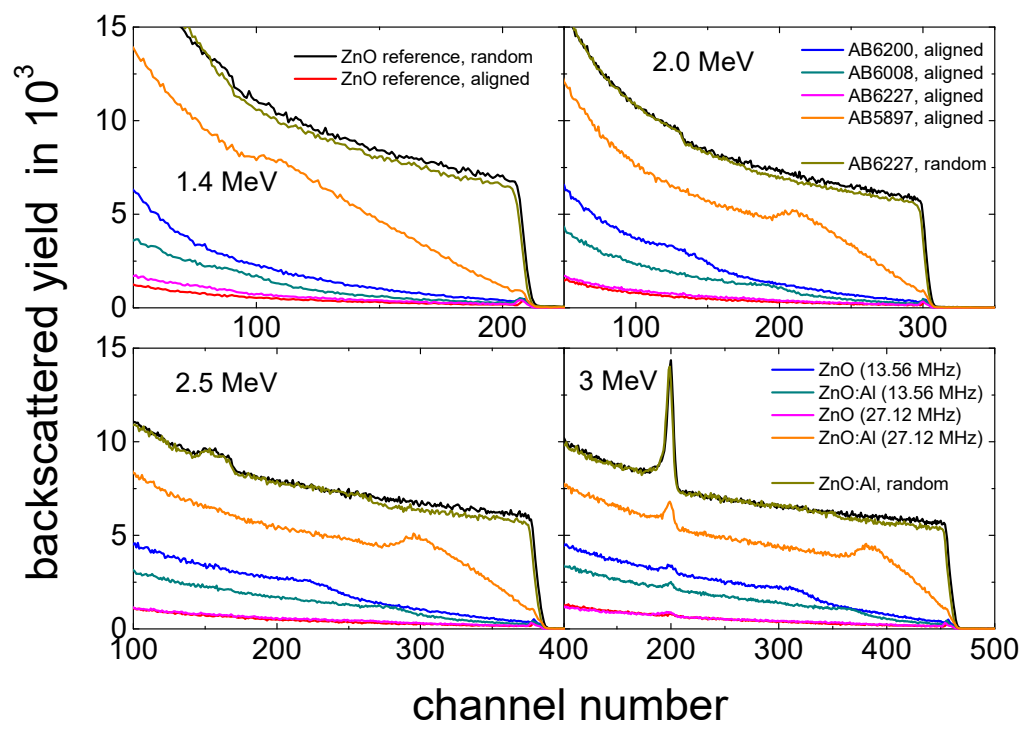

Figure 3. Energy spectra of He ions of different ion energies backscattered in homoepitaxial $\mathrm{ZnO}$ and $\mathrm{ZnO}: \mathrm{Al}$ layers fabricated under various sputtering conditions. Spectra in aligned and random directions for a $\mathrm{ZnO}$ single crystal are shown for comparison. Beam energies and names of the samples are indicated in the figure.

The minimum yield $x_{\min }$ is calculated as explained in Section 2 and shown in Figure 4 for all beam energies and each sample. It can be seen that in general the energy dependence of $\chi_{\min }$ is weak. The minimum yield $\chi_{\min }$ averaged over depths ranging from 600 to $650 \mathrm{~nm}$ was analysed with respect to its energy dependence according to $E^{K}$. The obtained values of $K$ are between -0.24 and +0.03 . According to theory, a value of $\mathrm{k} \approx 0.0$ points to the existence of stacking faults. However, in epitaxially grown layers one usually expects the existence of dislocations, which were indeed found in these homoepitaxial $\mathrm{ZnO}$ layers by transmission electron microscopy [7]. Therefore, one can assume that the weak energy dependence of the RBS minimum yield results from the superposition of point defects and dislocation loops both occurring in the sputtered layers. As described above, point defects result in a decrease in $\chi_{\min }$ with increasing beam energy and dislocations result in an increase, thus yielding a weak energy dependence in the case of superposition.

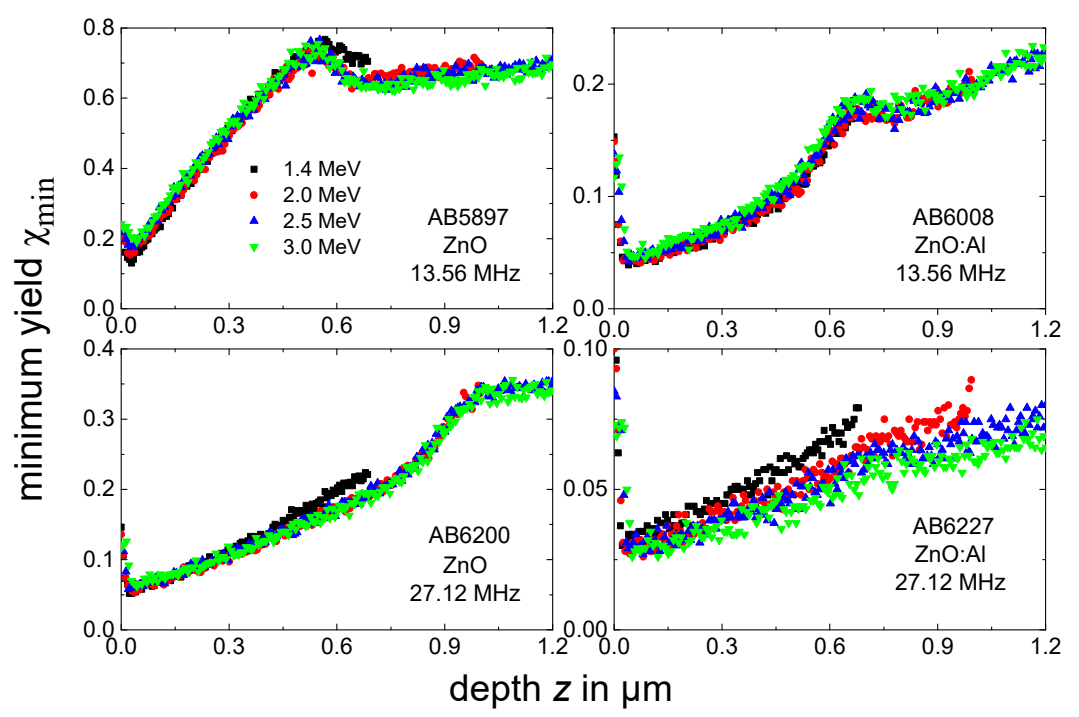

Figure 4. Minimum yield $\chi_{\min }$ versus depth $z$ for each sample and all of the He ion energies applied. The names of the samples are given in the figure. 
To demonstrate the effect of point defects and dislocation loops on the structure of the minimum yield $\chi_{\min }$ versus depth, the minimum yield for different defect distributions were calculated with DICADA1 (see Section 2). Figure 5 plots the results of the test calculations in comparison with the spectrum of minimum yield $\chi_{\min }$ for sample AB6008 ( $\mathrm{ZnO}: \mathrm{Al}$ deposited with $13.56 \mathrm{MHz}$ ) and analysis with $2 \mathrm{MeV}$ He ions. For the calculations shown in Figure 5a a box-like depth profile of randomly distributed displaced lattice atoms (representing point defects) was assumed with a thickness of $800 \mathrm{~nm}$ and various concentrations. The spectra in Figure $5 \mathrm{~b}$ were calculated assuming a box-like distribution of dislocation loops within the first $700 \mathrm{~nm}$ and various areal concentrations. A Burger's vector perpendicular to the loop plane and parallel to the channel direction was assumed. The magnitude of the Burger's vector was chosen to be $c=3.84 \AA$ and the loop radius $2500 \AA$. Figure 5 reveals that there are two significant differences between the two sets of calculated spectra. First, in the case of point defects, the calculated $\chi_{\min }$ spectra shift to higher values starting from the surface with increasing defect concentration. This is not the case for dislocation loops even though their concentration was assumed to be constant over depth up to the surface. Second, there is always a slight decrease in $\chi_{\min }$ at the end of the layer in the case of point defects. However, in the case of dislocation loops, the end of the layer is only indicated by a change in the slope of $\chi_{\min }$ versus depth.

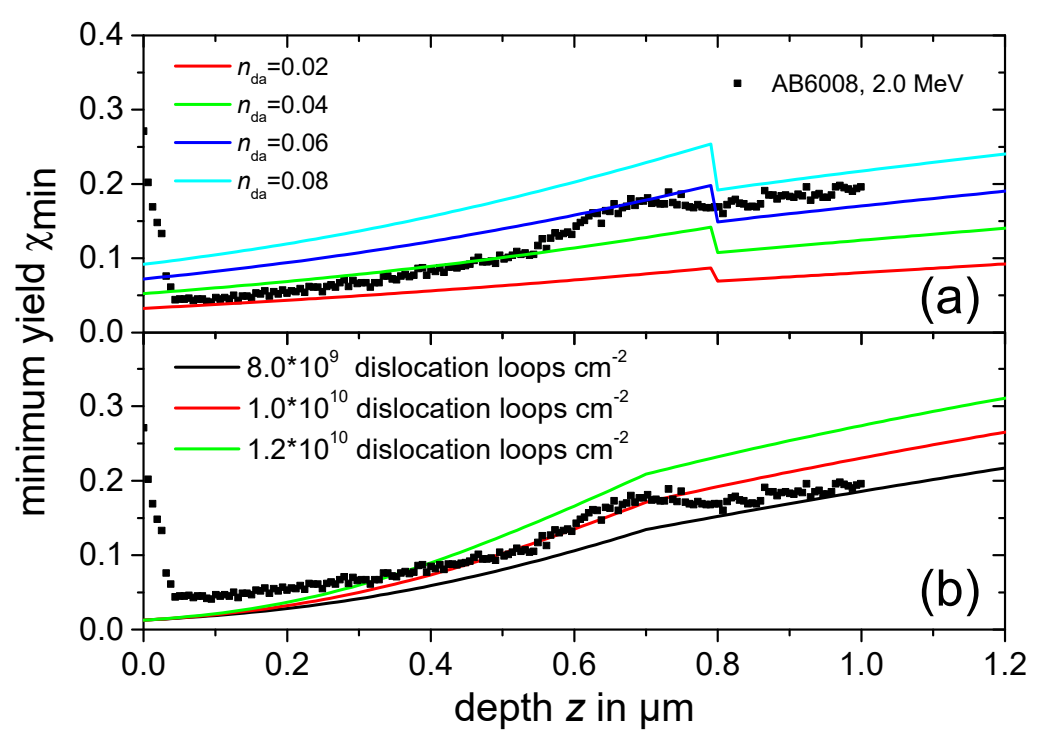

Figure 5. Minimum yield $\chi_{\text {min }}$ versus depth $z$ for sample AB6008 (ZnO:Al deposited with $13.56 \mathrm{MHz}$ ) measured with He ions of $2.0 \mathrm{MeV}$ energy (symbols). The impact of the different type and number of defects on the $\chi_{\min }$ spectra are demonstrated by DICADA1 calculations (solid lines), (a) shows the impact of point defects represented by randomly distributed displaced lattice atoms with various relative concentrations $n_{\mathrm{da}}$. In (b), spectra are depicted assuming dislocation loops of various areal concentrations. For details see text.

When comparing the measured $\chi_{\min }$ spectrum in Figure 5 with the calculated ones, it becomes obvious that both types of defects have to be taken into account to represent the measured spectra by calculated ones. In doing so, the contribution of dislocation loops is determined by the height of the minimum yield at depths behind the sputtered layer. Therefore, it is a precondition for this type of analysis that the DICADA code well describes the dechannelling background for point defects (i.e., uncorrelated-displaced lattice atoms). This is indeed the case, as was shown in previous publications [21,22]. Further, it has to be mentioned that information about the type and size of dislocation loops cannot be easily obtained with ion channelling [12]. However, the advantage is that, when assuming a certain type and size of loops, their contribution to the dechannelling of the analysing He ions can be quantified. This is useful when analysing a set of layers produced under different deposition conditions, as in this work. A similar procedure was applied for ion-implanted 
layers to study the number of point defects and dislocation loops as a function of the ion fluence by ion channelling [23,24].

Based on the results presented above, for each sample, the $\chi_{\min }$ spectra for all four beam energies applied were fitted by calculated $\chi_{\min }$ spectra assuming one and the same distribution of point defects and dislocation loops for all beam energies. The conditions for the corresponding DICADA1 calculations are as given above. The depth distributions of point defects and dislocation loops were varied until an optimum agreement between the calculated and measured spectra was obtained for all beam energies applied. The achieved fit of the spectra is demonstrated in Figure 6, which compares the measured and calculated spectra for the lowest $(1.4 \mathrm{MeV})$ and highest beam energy $(3 \mathrm{MeV})$. For all of the samples investigated, a good agreement was obtained. This also holds for the two medium beam energies (not shown). The resulting depth distribution of point defects and dislocation loops are plotted in Figure 7. The amount of both types of defects decreases with increasing deposition frequency. This is true for both materials, $\mathrm{ZnO}$ with and without $\mathrm{Al}$. This is in good agreement with former investigations [25], since higher deposition frequencies lead to lower voltage at the sputtering target. This discharge voltage determines the maximum energy of ions or energetic atoms, bombarding the growing film. Bombardment with higher-energy particles may cause more defects.

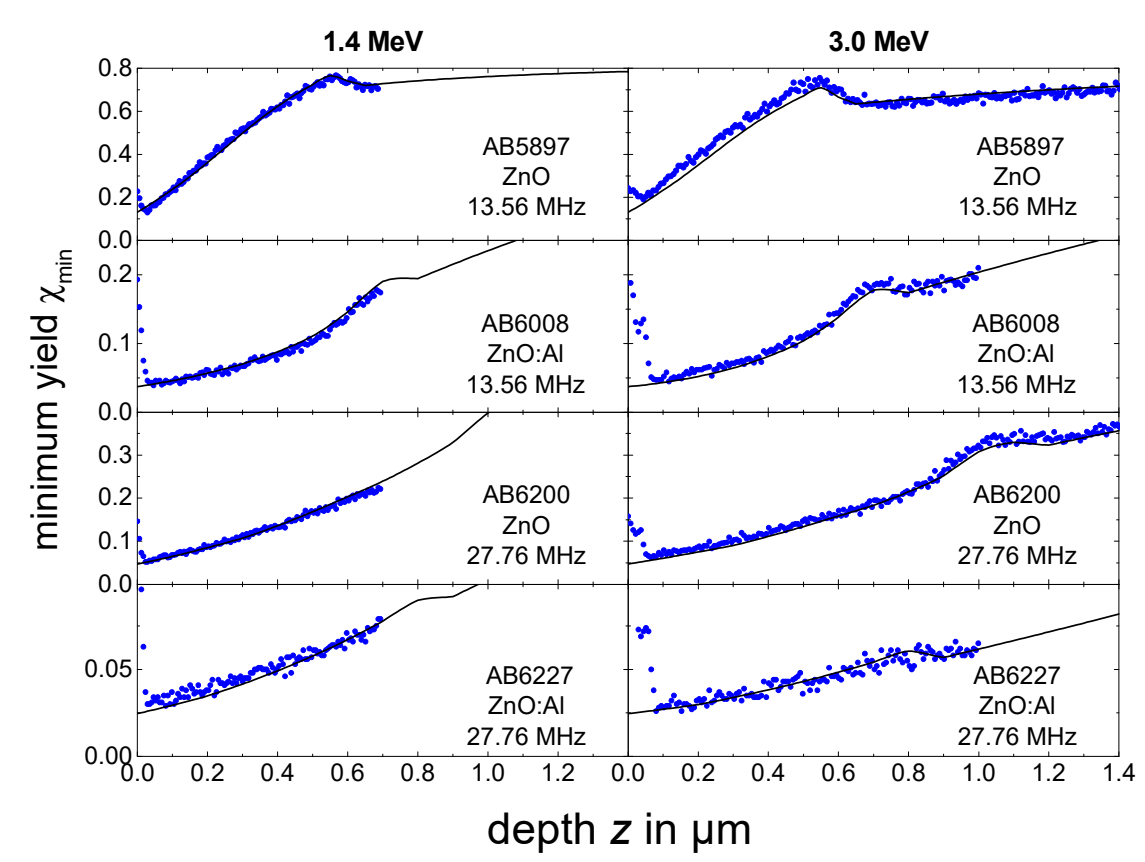

Figure 6. Measured (symbols) and calculated (solid lines) minimum yield $\chi_{\min }$ versus depth $z$ for He ion energies of $1.4 \mathrm{MeV}$ (left column) and 3.0 MeV (right column). The names of the samples are given in the figure.

Furthermore, the profiles generally show a lower amount of both types of defects for the layers containing Al. The reduction in the concentration of dislocation loops with increasing frequency is the same for both types of material, $\mathrm{ZnO}$ and $\mathrm{ZnO}$ :Al. The impact of the frequency on the reduction in the concentration of point defects is higher for samples containing no Al. One possible explanation is that the number of point defects is generally low in layers containing Al. 


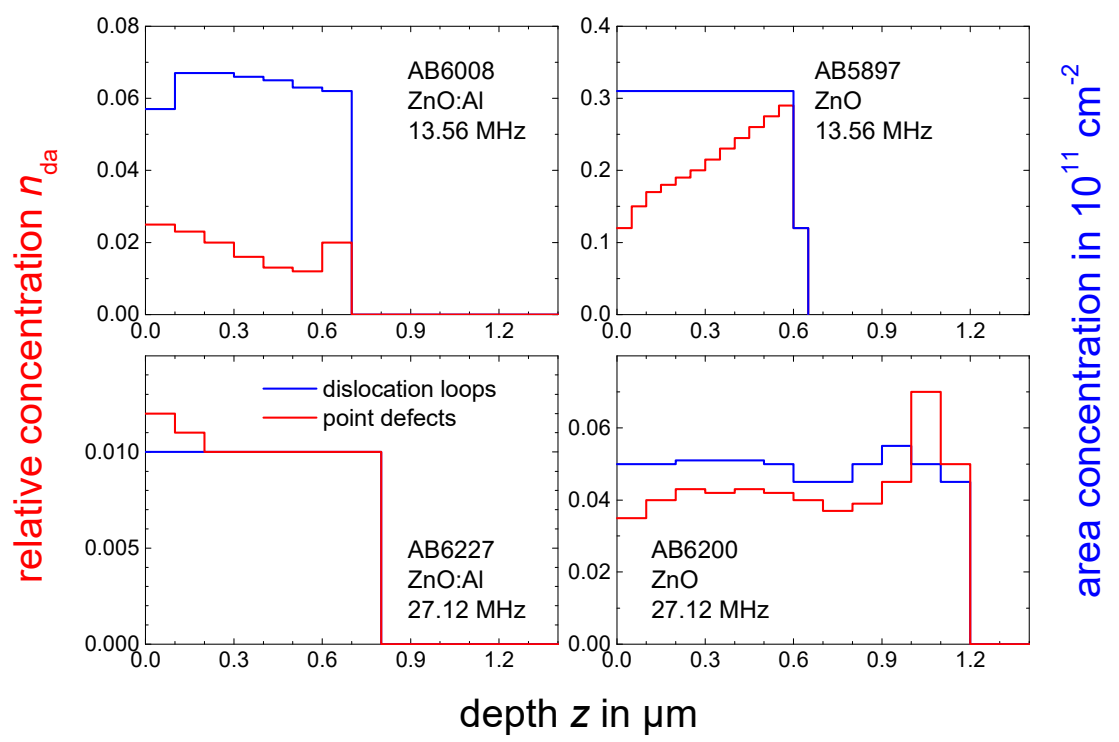

Figure 7. Depth distribution of the defect concentrations used to calculate the $\chi_{\min }$ spectra plotted in Figure 6 for the various homoepitaxial $\mathrm{ZnO}$ and $\mathrm{ZnO}: \mathrm{Al}$ layers investigated, which are indicated in the figure. Please note the different ordinate scales in different parts of the figure.

\section{Summary and Conclusions}

$\mathrm{ZnO}$ and $\mathrm{ZnO}: \mathrm{Al}$ were deposited on $<0001>\mathrm{ZnO}$ by RF-magnetron sputtering applying two different discharge frequencies. The crystalline quality of the deposited layers was investigated by energy-dependent RBS measurement in channelling configuration. For this, He ions with energies of $1.4,2.0,2.5$, and $3.0 \mathrm{MeV}$ were used. RBS analysis is quick, almost destruction free and does not require special sample preparation.

The minimum yield $\chi_{\min }$ of the backscattered ions measured as a function of the beam energy is sensitive to the type of defects. For all layers studied here, the $\chi_{\min }$ spectra measured with the ions of the different beam energies were well represented, assuming point defects and dislocation loops as the main type of defects in the layers. It is worth noting that an agreement was indeed possible using the same defect profiles (one for point defects and one for dislocation loops) for all beam energies. The existence of dislocation loops was proven by transmission electron microscopy in previous publications. The possibility of quantifying the number of point defects is a significant advantage of RBS. This information can be important for understanding, e.g., the electrical and optical properties of such layers.

Two main results can be deduced from our RBS studies: (1) The quantity of defects decreases with increasing RF frequency during the deposition. This holds well for both, $\mathrm{ZnO}$ and $\mathrm{ZnO}$ :Al layers; and (2) $\mathrm{ZnO}$ :Al layers are less damaged than those without $\mathrm{Al}$ for a given RF frequency. These results are in agreement with previous findings for similarly deposited layers investigated by other techniques. We were able to show the depth distribution of those two types of defects for both highly and lowly disturbed epitaxial layers.

Our study reveals that by exploiting the channelling effect and its energy dependence, more information can be obtained about the type and concentration of defects in crystals than by using only one ion energy. The procedure demonstrated here can be taken as an example that can also be applied to other defective crystals or thin crystalline layers.

Author Contributions: Conceptualization, F.W. and E.W.; Formal analysis, F.W. and K.G.; Funding acquisition, E.W. and K.E.; Investigation, F.W. and A.B.; Methodology, K.E. and E.W.; Project administration, K.E. and E.W.; Resources, A.B., K.E. and E.W.; Software, K.G.; Supervision, E.W.; Visualization, F.W. and E.W.; Writing-original draft, F.W. and E.W.; Writing-review \& editing, F.W., E.W., K.E. and K.G. 
Acknowledgments: The work was supported by the Bundesministerium für Bildung und Forschung of the Federal Republic of Germany under contract number 03SF0478B and by the German Federal Ministry of Economics and Technology (Grant Nos. KF2002606DA0 and AiF 16595BG).

Conflicts of Interest: The authors declare no conflict of interest.

\section{References}

1. Cebulla, R.; Wendt, R.; Ellmer, K. Al-doped zinc oxide films deposited by simultaneous rf and dc excitation of a magnetron plasma: Relationships between plasma parameters and structural and electrical film properties. J. Appl. Phys. 1998, 83, 1087-1095. [CrossRef]

2. Özgür, Ü.; Alivov, Y.I.; Liu, C.; Teke, A.; Reshchikov, M.A.; Doğan, S.; Avrutin, V.; Cho, S.J.; Morkoç, H. A comprehensive review of $\mathrm{ZnO}$ materials and devices. J. Appl. Phys. 2005, 98, 11. [CrossRef]

3. Greene, J.E. Tracing the recorded history of thin-film sputter deposition: From the1800s to 2017. J. Vac. Sci. Technol. A 2017, 35, 05C204. [CrossRef]

4. Chu, W.K.; Mayer, J.W.; Nicolet, M.A. Backscattering Spectrometry; Academic Press: New York, NY, USA, 1978.

5. Götz, G.; Gärtner, K. (Eds.) High Energy Ion Beam Analysis; Akademie-Verlag: Berlin, Germany, 1988.

6. Wesch, W.; Wendler, E. (Eds.) Ion Beam Modification of Solids: Ion-Solid Interaction and Radiation Damage; Springer International Publishing: Cham, Switzerland, 2016.

7. Rengachari, M.; Bikowski, A.; Ellmer, K. Defect analysis by transmission electron microscopy of epitaxial Al-doped $\mathrm{ZnO}$ films grown on (0001) ZnO and a-sapphire by RF magnetron sputtering. J. Appl. Phys. 2016, 120, 015305. [CrossRef]

8. Bikowski, A.; Rengachari, M.; Nie, M.; Wanderka, N.; Stender, P.; Schmitz, G.; Ellmer, K. Research Update: Inhomogeneous aluminium dopant distribution in magnetron sputtered $\mathrm{ZnO}: \mathrm{Al}$ thin films and its influence on their electrical properties. Appl. Phys. Lett. Mater. 2015, 3, 060701. [CrossRef]

9. Nie, M.; Bikowski, A.; Ellmer, K. Microstructure evolution of Al-doped zinc oxide and Sn-doped indium oxide deposited by radio-frequency magnetron sputtering: A comparison. J. Appl. Phys. 2015, 117, 155301. [CrossRef]

10. Ziegler, J.F.; Biersack, J.P.; Littmark, U. The Stopping and Ranges of Ions in Solids; Pergamon: New York, NY, USA, 2003.

11. Gärtner, K. Modified master equation approach of axial dechannelling in perfect compound crystals. Nucl. Instrum. Methods Phys. Res. B 2005, 227, 522-530. [CrossRef]

12. Gärtner, K.; Ugguzoni, A. Energy dependence of dechannelling due to dislocation loops. Nucl. Instrum. Methods Phys. Res. B 1992, 67, 189-193. [CrossRef]

13. Gärtner, K.; Hehl, H.; Schlotzhauer, G. AXIAL DECHANNELLING: I. Perfect Crystal. Nucl. Instrum. Methods 1983, 216, 275-286. [CrossRef]

14. Gärtner, K.; Hehl, H.; Schlotzhauer, G. AXIAL DECHANNELLING: II. Point Defects. Nucl. Instrum. Methods B 1984, 4, 55-62. [CrossRef]

15. Friedland, E.; Gärtner, K.; Hlatshwayo, T.T.; van der Berg, N.G.; Thabethe, T.T. Influence of radiation damage on xenon diffusion in silicon carbide. Nucl. Instrum. Methods Phys. Res. B 2014, 332, 415-420. [CrossRef]

16. Robie, R.A.; Edwards, J.L. Some Debye temperatures from single-crystal elastic constant data. J. Appl. Phys. 1966, 37, 2659-2663. [CrossRef]

17. Wendler, E.; Bilani, O.; Gärtner, K.; Wesch, W.; Hayes, M.; Auret, F.D.; Lorenz, K.; Alves, E. Radiation damage in ZnO ion implanted at $15 \mathrm{~K}$. Nucl. Instrum. Methods Phys. Res. B 2009, 267, 2708-2711. [CrossRef]

18. Wendler, E.; Treiber, E.; Baldauf, J.; Wolf, S.; Ronning, C. High-level damage saturation below amorphisation in ion implanted $\beta-\mathrm{Ga}_{2} \mathrm{O}_{3}$. Nucl. Instrum. Methods Phys. Res. B 2016, 379, 85-90. [CrossRef]

19. Gruska, B.; Götz, G. Dechannelling by dislocations in ion implanted Si. Radiat. Effects 1982, 59, $157-167$. [CrossRef]

20. IBANDL (Ion Beam Analysis Nuclear Data Library). Available online: https://www-nds.iaea.org/exfor/ ibandl.htm (accessed on 13 May 2019).

21. Gärtner, K. Axial dechannelling in compound crystals with point defects and defect analysis by RBS. Nucl. Instrum. Methods Phys. Res. B 1997, 132, 147-158. [CrossRef]

22. Wendler, E.; Gaiduk, P.I. Rutherford backscattering analysis of damage in ion implanted GaAs/A1 $1_{0.44} \mathrm{Ga}_{0.56} \mathrm{As}$. Nucl. Instrum. Methods Phys. Res. B 1998, 136, 488-493. [CrossRef] 
23. Turos, A. On the mechanism of damage buildup in gallium nitride. Radiat. Eff. Defects Solids 2013, 168, 431-441. [CrossRef]

24. Haddad, Y.; Delauche, L.; Gentils, A.; Garrido, F. In situ characterization of irradiation-induced microstructural evolution in urania single crystals at 773 K. Nucl. Instrum. Methods Phys. Res. B 2018, 435, 25-30. [CrossRef]

25. Bikowski, A.; Welzel, T.; Ellmer, K. The impact of negative oxygen ion bombardment on electronic and structural properties of magnetron sputtered ZnO:Al films. Appl. Phys. Lett. 2013, 102, 242106. [CrossRef]

(C) 2019 by the authors. Licensee MDPI, Basel, Switzerland. This article is an open access article distributed under the terms and conditions of the Creative Commons Attribution (CC BY) license (http://creativecommons.org/licenses/by/4.0/). 